\title{
Expression and function of cystic fibrosis transmembrane conductance regulator in rat intrapulmonary arteries
}

\author{
R. Robert*, J-P. Savineau", ${ }^{\#,}$ C. Norez*, F. Becq* and C. Guibert ${ }^{\#, 9}$
}

ABSTRACT: The cystic fibrosis transmembrane conductance regulator (CFTR) gene encodes a cyclic adenosine monophosphate (cAMP)-dependent chloride channel located mainly at the apical membrane of epithelial cells. In myocytes of pulmonary arteries, numerous chloride channels have been identified and described, but not the CFTR. Thus the presence and function of the CFTR was investigated in rat intrapulmonary arteries.

CFTR expression, localisation and function were analysed in cultured smooth muscle cells using Reverse transcriptase (RT)-PCR and immunoprecipitation followed by protein kinase A phosphorylation, immunolocalisation and an iodide efflux assay, respectively. The role of the CFTR in pulmonary vasoreactivity was determined in arterial rings using an organ bath system.

RT-PCR and immunoprecipitation analyses, as well as the immunolocalisation study, revealed the expression of CFTR gene transcripts and protein. The iodide efflux assay showed the existence of functional cAMP-, calcium- and volume-dependent chloride channels. Furthermore, the following effects were found: 1) inhibition of forskolin/genistein-activated iodide efflux by glibenclamide, diphenylamine-2-carboxylic acid and CFTR-specific inhibitor (CFTRinh)-172; 2) activation of iodide efflux by the benzoquinolizinium derivative CFTR activators MPB-07 and MPB91; and 3) inhibition of MPB-dependent efflux by CFTRinh-172. Finally, CFTR activators induced concentration-dependent vasorelaxation in rings preconstricted with phenylephrine, in the presence or absence of endothelium.

The present results are the first to reveal functional cyclic adenosine monophosphate-regulated cystic fibrosis transmembrane conductance regulator contributing to endothelium-independent vasorelaxation in rat intrapulmonary arterial myocytes.

KEYWORDS: Chloride channels, cystic fibrosis transmembrane conductance regulator, intrapulmonary arteries, iodide efflux, smooth muscle cells, vasoreactivity

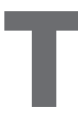
he cystic fibrosis transmembrane conductance regulator (CFTR) is a cyclic adenosine monophosphate (cAMP)-dependent chloride channel expressed at the apical membrane of epithelial cells lining the tracheobronchial tree and the lumen of the digestive tract [1]. The CFTR was generally regarded as specifically expressed in epithelial cells until evidence for CFTR expression in nonepithelial tissues emerged. CFTR is expressed in cardiac muscle cells [2], brain [3] and endothelia [4], and has recently been found in tracheal smooth muscle cells (SMCs) [5] and aortic SMCs of rats and mice $[6,7]$. Despite this CFTR expression profile, the clinical picture of patients suffering from the CFTR-related disease cystic fibrosis appears to be unrelated to cardiac, vascular and brain dysfunction. Since truly selective activators and inhibitors are also lacking, the role of the CFTR in these tissues remains unresolved. However, the pharmacology of the CFTR has recently progressed, making available several CFTR activaderivatives [6-8], as well as blockers, such as the thiazolidinone compound CFTR-specific inhibitor 3-((3-trifluoromethyl)phenyl)-5-((4-carboxyphenyl) methylene)-2-thioxo-4-thiazolidinone (CFTRinh172) [9].

Several $\mathrm{Cl}^{-}$conductances have been described in smooth muscle, including the pulmonary artery. Among the $\mathrm{Cl}^{-}$channels expressed in SMCs, extensive studies have explored the implication of calcium-activated $\mathrm{Cl}^{-}$currents $(\mathrm{ICl}, \mathrm{Ca})$, evoked by a rise in intracellular calcium concentration $\left(\left[\mathrm{Ca}^{2+}\right]_{\mathrm{i}}\right)[10]$. Although the molecular nature of tors, such as genistein and benzoquinolizinium

\section{AFFILIATIONS}

*Institute of Cellular Physiology and Biology, UMR 6187, National Centre for Scientific Research (CNRS), University of Poitiers, Poitiers, and ${ }^{\#}$ Laboratory of Respiratory Cellular Physiology, University of Bordeaux 2, 33076, Bordeaux. •Inserm U885, 33076 Bordeaux, France.

CORRESPONDENCE

C. Guibert

INSERM U885

Laboratoire de Physiologie Cellulaire Respiratoire

146 rue Léo Saignat F33076 Bordeaux France

Fax: 33557571695

E-mail: christelle.guibert@ubordeaux2.fr

Received

May 162007

Accepted after revision:

June 202007

\section{SUPPORT STATEMENT}

None declared.This study was supported by Overcoming Cystic Fibrosis (VLM; Paris, France). R. Robert and C. Norez were supported by a studentship from VLM.

STATEMENT OF INTEREST None declared. 
the $\mathrm{ICl}, \mathrm{Ca}$ is still unknown, its role in resting membrane potential and vascular tone has been described for pulmonary artery [11-13].

Regarding the volume-sensitive $\mathrm{Cl}^{-}$channel (ICl,swell), members of the $\mathrm{Cl}^{-}$channel $(\mathrm{ClC})$ gene family appear to be good molecular candidates for ICl,swell, and ClC-3 was recently proposed to underlie $\mathrm{ICl}$,swell in rat and canine pulmonary arteries [14]. Interestingly, $\mathrm{ClC}-3$ is upregulated in pulmonary hypertensive rats [14]. It is also noteworthy that the contribution of $\mathrm{Cl}^{-}$channels is enhanced in basal tone and norepinephrine-induced contraction in pulmonary hypertensive rats $[15,16]$.

A cAMP-dependent $\mathrm{Cl}^{-}$channel has also been described in rat and bovine pulmonary arteries [17, 18]. This channel is implicated in cAMP-induced pulmonary vasodilation, pulmonary arterial SMC (PASMC) migration and morphological changes. However, the molecular identity of such a channel is still unknown in pulmonary artery and could be related to the CFTR.

Despite the fact that the CFTR is present in aortic SMCs [6, 7], and that $\mathrm{Cl}^{-}$channels are important factors in the physiology as well as in the pathophysiology of the pulmonary artery, no studies have investigated the expression and functional role of the CFTR in pulmonary artery to date. Consequently, the present study focused on intrapulmonary artery (IPA) and investigated the expression of the CFTR in this vessel, and then explored cAMP-dependent iodide efflux, its pharmacology and the effect of CFTR activators on pulmonary vasorelaxation.

\section{METHODS}

\section{Tissue preparation}

Male Wistar rats (aged 8-10 weeks) (Janvier, Le Genest-SaintIsle, France) were stunned and then killed by cervical dislocation according to the local animal care and use committee (agreement number AP2/11/2005 from the regional ethics committee of Aquitaine/Poitou-Charentes, France). The heart and lungs were removed and placed in Krebs-Henseleit (KH) solution, which comprised $118.4 \mathrm{mM} \mathrm{NaCl}, 4.7 \mathrm{mM} \mathrm{KCl}$,

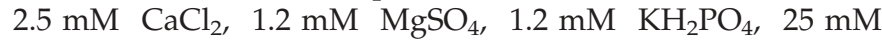
$\mathrm{NaHCO}_{3}$ and $11.1 \mathrm{mM}$ D-glucose $(\mathrm{pH} 7.4)$, saturated with $15 \%$ oxygen $/ 5 \%$ carbon dioxide $/ 80 \%$ nitrogen. IPA of the first order from the left lung was dissected free from surrounding connective tissues in $\mathrm{KH}$ solution.

\section{Cell culture}

The entire heart-lung preparation was rapidly removed and rinsed in culture medium (Dulbecco's modified Eagle medium/HEPES ( $\mathrm{pH}$ 7.3) supplemented with $1 \%$ penicillin-streptomycin, $1 \%$ sodium pyruvate and $1 \%$ nonessential amino acids). IPA was dissected in culture medium under sterile conditions and cut into several pieces $\left(1-2 \mathrm{~mm}^{2}\right)$. SMCs were obtained from these explants and cultured as previously described [5]. All cells from these explants were immunostained using the monoclonal antibody anti-smooth muscle $\alpha$ actin (Sigma-Aldrich, Saint-Quentin Fallavier, France), whereas they were negatively labelled with the endothelial nitric oxide synthase antibody (BD Transduction Laboratories, Le Pont-de-Claix, France; data not shown), demonstrating the presence of a population of SMCs.

\section{Analysis of CFTR mRNA expression by reverse transcriptase-PCR}

Total RNA was extracted using $\mathrm{RNABI}_{\mathbb{B}}$ (Eurobio, Courtaboeuf, France), according to the manufacturer's protocol. Complementary DNA was used as template in PCRs with PCR primers specific for the rat CFTR gene [19]: cfrex3 (5'GCGATGCTTTGTCTGGAGATTC-3'), and cfrex6a (5'-CCACTTGTAAAGGAGCAATCCATA-3'), spanning the region between nucleotides 222 and 625. The housekeeping $\beta$-actin gene (GenBank accession No. NM031144) was used as control, with the primers r-acti4 (5'-CTACCTCATGAAGATCCTGA$\left.3^{\prime}\right)$ and r-acti5 (5'-TTTCATGGATGCCACAGGAT-3'), spanning the region between nucleotides 561 and 829 . The primers were checked for matches in separate exons, by aligning their sequences with rat genomic sequences.

\section{Immunoprecipitation and phosphorylation of CFTR}

Chinese hamster ovary $(\mathrm{CHO})$ cells stably expressing wildtype CFTR (used as a positive control for CFTR expression) and IPA SMCs were washed three times in PBS ( $\mathrm{pH} 7.4)$, scraped in radioimmunoprecipitation assay buffer $(50 \mathrm{mM}$ tris(hydroxymethyl)aminomethane (Tris)- $\mathrm{HCl}$ ( $\mathrm{pH}$ 7.5), $1 \mathrm{mM}$ ethylenediamine tetra-acetic acid (EDTA), $100 \mathrm{mM} \mathrm{NaCl}$ and $1 \%$ Triton X-100) supplemented with protease inhibitors (20 $\mu \mathrm{M}$ leupeptin, $0.8 \mu \mathrm{M}$ aprotinin, $10 \mu \mathrm{M}$ pepstatin and $2.1 \mathrm{mM}$ 4-(2-aminoethyl)-benzenesulphonyl fluoride hydrochloride) and homogenised by several passes through a 23gauge syringe needle. Cell lysates were then treated as previously described [7]. Briefly, lysates were incubated with CFTR mouse monoclonal antibody clone 24-1 (R\&D Systems, Minneapolis, MN, USA) or nonimmune mouse immunoglobulin (Ig) G (Sigma-Aldrich). The CFTR was phosphorylated in vitro using two units of the catalytic subunit of protein kinase (PK)A (Sigma-Aldrich) and $370 \mathrm{kBq}\left[\gamma_{-}{ }^{32} \mathrm{P}\right]$ adenosine triphosphate (ATP; Amersham Pharmacia Biotech, Orsay, France; $\left.111 \mathrm{TBq} \cdot \mathrm{mmol}^{-1}\right)$. Phosphorylated proteins were visualised by autoradiography.

\section{Immunofluorescence study}

Immunofluorescent labelling of IPA SMCs using an anti-CFTR C-terminus monoclonal antibody (mouse anti-human clone 241; R\&D Systems) with TO-PRO-3 iodide (Molecular Probes, Invitrogen Corporation, Cergy-Pontoise, France) for nuclear staining was performed as previously described [5].

\section{Isometric tension measurements}

The effect of CFTR activators on mechanical activity were measured using IPA rings (1.5-2.5 mm long) as previously reported [20]. In brief, KH solution, saturated with $15 \%$ oxygen/ $5 \%$ carbon dioxide $/ 80 \%$ nitrogen, was used. Mechanical properties were assessed using an organ bath and transducer systems (EMKA Technologie, Paris, France), coupled to IOX software (EMKA Technologie) in order to facilitate data acquisition and analysis. As determined in preliminary experiments, tissues were set at optimal length by equilibration against a passive load of $0.8 \mathrm{~g}$. IPA rings were then washed and precontracted with $0.3 \mu \mathrm{M}$ phenylephrine (PHE) in order to test the relaxant properties of CFTR activators (10-chloro-6hydroxybenzo[c]quinolizinium chloride (MPB-07), 5-butyl-10chloro-6-hydroxybenzo[c]quinolizinium chloride (MPB-91) and 10-fluoro-6-hydroxybenzo[c]quinolizinium chloride (MPB-80); 
3-300 $\mu \mathrm{M}$, prepared as described previously [21]) by constructing a cumulative concentration-response curve. Some experiments were performed in endothelium-denuded rings. Endothelium was removed by perfusing the lumen of the vessels with a solution containing $0.3 \%$ 3-((3-cholamidopropyl) diethylammonio)-1-propane sulphonate (CHAPS), followed by washout with the drug-free solution, as previously described [22]. The effect of CHAPS was confirmed by the absence of relaxation with $10 \mu \mathrm{M}$ carbamylcholine of $0.3 \mu \mathrm{M}$ PHE-induced precontraction. All experiments were performed at $37^{\circ} \mathrm{C}$.

All chemical agents were dissolved in dimethylsulphoxide (DMSO; final concentration $\leqslant 0.1 \%$ ), except $5,11,17,23-$ tetrasulphonato-25,26,27,28-tetramethoxy-calix[4]arene (calixarene; generously provided by A.K. Singh and R.J. Bridges (University of Pittsburgh, Pittsburgh, PA, USA)), carbamylcholine, CHAPS, MPB-07, MPB-80 and PHE, which were dissolved in water. The maximal concentration of DMSO used in the experiments was $\leqslant 0.1 \%$ for all of the chemicals and $0.3 \%$ for MPB-91 and had no effect on the mechanical activity of the rings.

\section{lodide efflux}

CFTR $\mathrm{Cl}^{-}$channel activity was assayed by measuring the rate of ${ }^{125}$ I efflux from cultured cells as previously described [6, 7]. Cells were washed with efflux buffer, which comprised $136.9 \mathrm{mM} \mathrm{NaCl}, 5.4 \mathrm{mM} \mathrm{KCl}, 0.3 \mathrm{mM} \mathrm{KH}_{2} \mathrm{PO}_{4}, 0.3 \mathrm{mM}$ $\mathrm{NaH}_{2} \mathrm{PO}_{4}, 1.3 \mathrm{mM} \mathrm{CaCl}, 0.5 \mathrm{mM} \mathrm{MgCl}_{2}, 0.4 \mathrm{mM} \mathrm{MgSO}$, $5.6 \mathrm{mM}$ glucose and $10 \mathrm{mM}$ HEPES (pH 7.4).

In order to stimulate volume-sensitive $\mathrm{Cl}^{-}$transport, the osmolarity of the efflux buffer was reduced from 300 to $150 \mathrm{mOsm} \cdot \mathrm{L}^{-1}$. Cells incubated in efflux buffer containing $\mathrm{Na}^{125} \mathrm{I}$ (New England Nuclear, Boston, MA, USA; $37 \mathrm{kBq} \cdot \mathrm{mL}^{-1}$ ) for $1 \mathrm{~h}$ at $37^{\circ} \mathrm{C}$ were then washed with efflux medium in the presence or absence of $0.3 \mu \mathrm{M}$ PHE to remove extracellular ${ }^{125} \mathrm{I}$. Loss of intracellular ${ }^{125} \mathrm{I}$ was determined by removing the medium with the efflux buffer every minute for up to $8 \mathrm{~min}$. The first three aliquots were used to establish a stable baseline in efflux buffer alone. Medium containing the appropriate drug was used for the remaining aliquots. The fraction of the initial intracellular ${ }^{125} \mathrm{I}$ lost at each time-point was determined, and time-dependent rates of ${ }^{125} \mathrm{I}$ efflux were calculated thus:

$$
\ln \left({ }^{125} \mathrm{I} t_{1} /{ }^{125} \mathrm{I} t_{2}\right) /\left(t_{1}-t_{2}\right)
$$

where ${ }^{125} \mathrm{I} t$ is the intracellular ${ }^{125} \mathrm{I}$ concentration at time $t$, and $t_{1}$ and $t_{2}$ are successive time-points. Curves were constructed by plotting the rate of ${ }^{125}$ I efflux versus time. All comparisons were based on the maximal time-dependent rate $\left(k\right.$; in $\left.\min ^{-1}\right)$, excluding the points used to establish the baseline (kpeakkbasal).

\section{Statistical analysis}

Data are presented as the mean \pm SEM of $n$ observations, or the number of rings for the tension recordings. Sets of data were compared using ANOVA or an unpaired t-test. Differences were considered significant when $\mathrm{p}<0.05$.

\section{RESULTS}

\section{Expression of CFTR in IPA}

CFTR expression in IPA was revealed using three experimental approaches. First, the presence of CFTR mRNA was detected by reverse transcriptase-PCR (fig. 1a) in intestine, an organ known to express CFTR and used as a positive control for expression of the CFTR (lane 1), and PASMCs (lane 3), but not in rat skeletal muscle (lane 2). Secondly, immunoprecipitation using the anti-CFTR antibody followed by in vitro PKA phosphorylation analysis demonstrated that, as in the $\mathrm{CHO}$ cell line used as a positive control, mature CFTR was phosphorylated in vitro by PKA in cultured PASMCs (fig. 1b). The major CFTR form (band C) was a 175-kDa protein, as determined using molecular mass standards. Controls with nonimmune mouse IgG are also shown (lanes 2 and 4). Thirdly, an immunocytochemical approach was used to determine the presence and location of the CFTR in PASMCs. Cells were stained with the anti-CFTR C-terminus monoclonal antibody and anti-smooth muscle actin antibody, whereas no staining could be detected in control experiments (fig. 2). Taken together, these results demonstrate that the
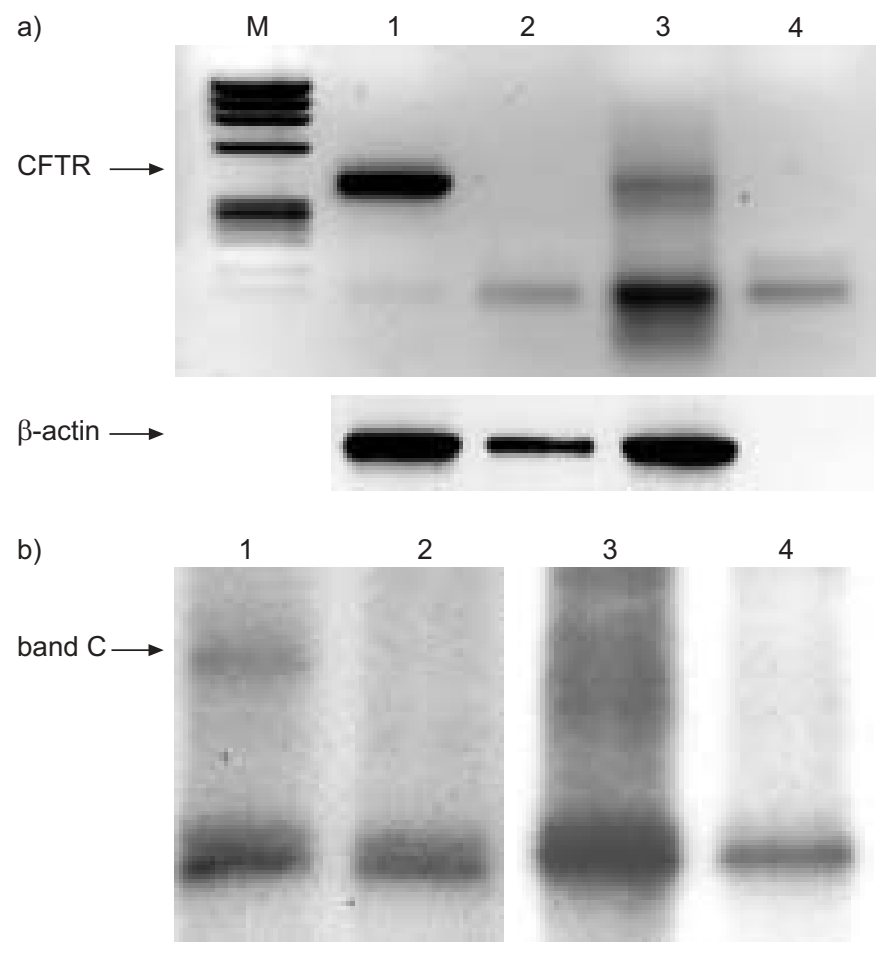

FIGURE 1. Expression of the cystic fibrosis transmembrane conductance regulator (CFTR) in cultured smooth muscle cells (SMCs) from rat intrapulmonary arteries (IPAs). a) Reverse transcriptase-PCR analysis of CFTR mRNA (403 bp) expression in rat intestine (lane 1), skeletal muscle (lane 2) and isolated pulmonary arterial SMCs (PASMCs; lane 3). Lane 4: no cDNA (water control). The expected size of the $\beta$-actin housekeeping gene was $269 \mathrm{bp}$. CFTR mRNA was expressed in rat intestine and isolated PASMCs but not in skeletal muscle. b) The CFTR (band C) was identified by immunoprecipitation followed by in vitro cyclic adenosine monophosphate-dependent protein kinase A phosphorylation in Chinese hamster ovary cells stably expressing wild-type CFTR (lanes 1 (positive control) and 2) and IPA SMCs (lanes 3 and 4). Lanes 2 and 4: CFTR antibody omitted (negative controls). This experiment was performed in duplicate. M: molecular mass standards. 

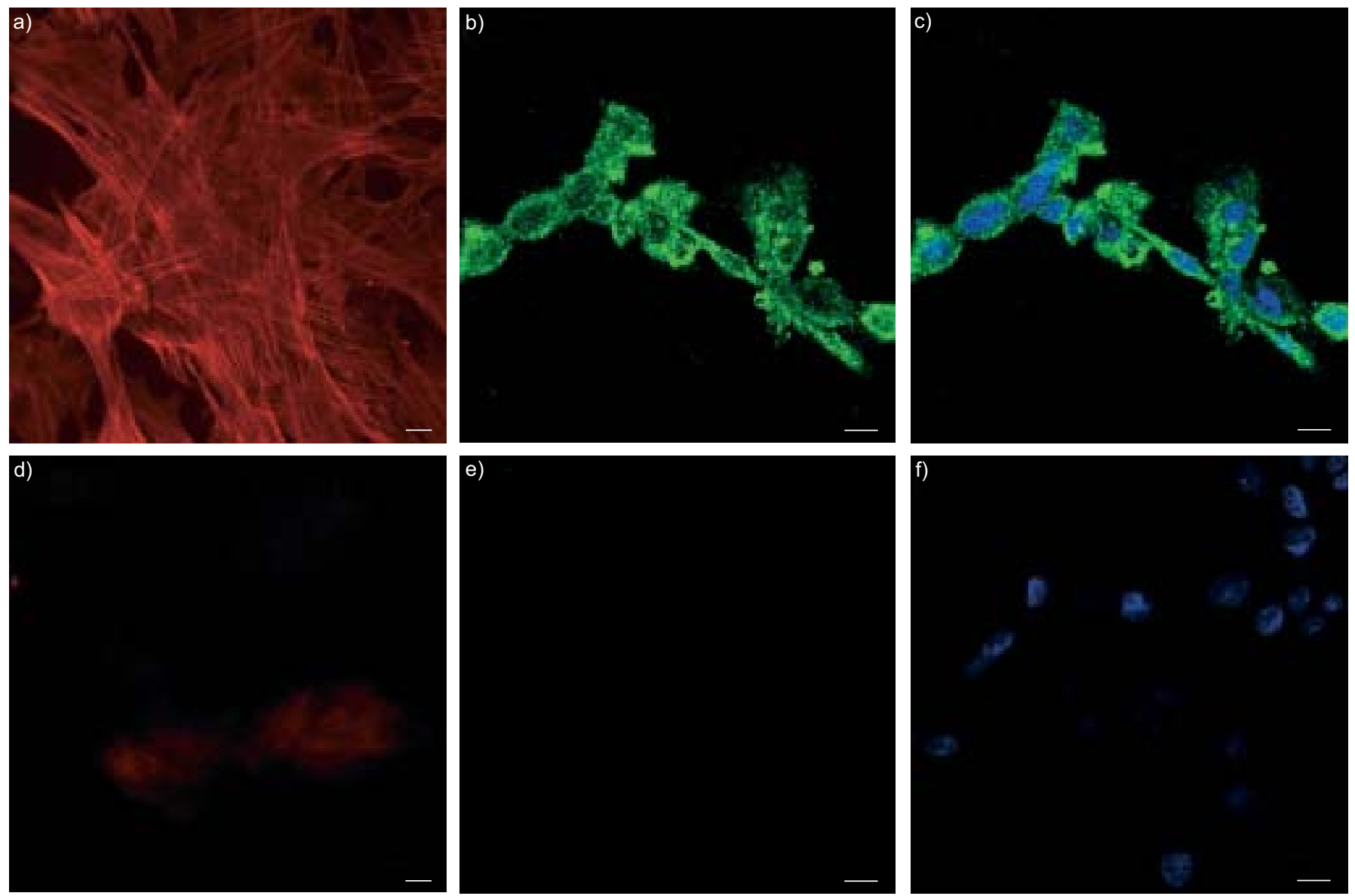

FIGURE 2. Immunolocalisation of the cystic fibrosis transmembrane conductance regulator (CFTR) in cultured smooth muscle cells from rat intrapulmonary arteries Confocal microscopic images showing: a) smooth muscle $\alpha$-actin staining (red; Cy3 was used as the secondary antibody); b, c) CFTR staining using anti-CFTR C-terminus monoclonal antibody (green); and c, f) nuclei labelled in blue with TO-PRO-3 iodide. Negative controls (d, e) were obtained by using the secondary antibody without the primary antibody. The merged images (c and f) show both nuclei and CFTR fluorescence. Scale bars $=10 \mu \mathrm{m}$.

CFTR is endogenously expressed in IPA SMCs and can be detected as PKA-phosphorylated mature protein.

\section{Analysis of chloride transport in cultured myocytes from IPA}

In order to verify that the iodide efflux method can be applied to IPA cultured cells, the cells were first exposed to hypoosmotic bath solution $\left(150 \mathrm{mOsm} \cdot \mathrm{L}^{-1}\right)$ to stimulate the endogenous ICl,swell (fig. 3a). The cAMP agonists vasoactive intestinal peptide $(500 \mathrm{nM})$ and forskolin (LC laboratory, PKC Pharmaceuticals, Inc., Woburn, MA, USA; $10 \mu \mathrm{M})$ plus the isoflavone genistein $(30 \mu \mathrm{M})$ and agents that raise $\left[\mathrm{Ca}^{2+}\right]_{\mathrm{i}}$, such as the $\mathrm{Ca}^{2+}$ ionophore A23187 $(1 \mu \mathrm{M})$ and ATP $(100 \mu \mathrm{M})$, significantly stimulated iodide efflux $(p<0.001 ; n=4)$, demonstrating the functional presence of multiple $\mathrm{Cl}^{-}$transporters dependent on cAMP, calcium and/or cell volume in rat IPA (fig. 3).

\section{Inhibitors of CAMP-dependent chloride transport in cultured intrapulmonary arterial myocytes}

Since the CFTR is activated by CAMP, it was hypothesised that the cAMP-dependent forskolin/genistein-activated $\mathrm{Cl}^{-}$transport would be supported by the CFTR. Glibenclamide and diphenylamine-2-carboxylic acid (DPC) are two nonspecific inhibitors of $\mathrm{Cl}^{-}$channels, including the CFTR [23], the stilbene derivative 4,4'-diisothiocyanatostilbene-2,2'-disulphonic acid (DIDS) is a nonspecific blocker of $\mathrm{Cl}^{-}$channels but does not inhibit the CFTR from the extracellular side of the plasma membrane [23], and calixarene is an inhibitor of outwardly rectifying $\mathrm{Cl}^{-}$channels but not of the CFTR [23, 24]. Forskolin/ genistein-dependent iodide efflux was fully inhibited by $100 \mu \mathrm{M}$ glibenclamide and $500 \mu \mathrm{M} \mathrm{DPC}$, but by neither $100 \mathrm{nM}$ calixarene nor $500 \mu \mathrm{M}$ DIDS ( $\mathrm{n}=4$ for each; fig. 4,). CFTRinh-172 (Calbiochem, VWR International, Fontenay-sous-Bois, France; $10 \mu \mathrm{M})$, a specific CFTR blocker [9], also strongly inhibited the iodide efflux response to forskolin/genistein (fig. 4), suggesting that the CFTR is likely to be responsible for the forskolin/ genistein-activated $\mathrm{Cl}^{-}$transport in cultured IPA cells.

\section{Role of CFTR in intrapulmonary arterial vasorelaxation}

Since cAMP-related agonists induce pulmonary vasorelaxation $[25,26]$, the effect of CFTR activators were investigated in rat IPA rings preconstricted with $0.3 \mu \mathrm{M}$ PHE. MPB-07 and -91 are good specific CFTR activators, whereas MPB-80 is a very poor CFTR activator but a useful control $[6,7,21]$. Unlike MPB-80, MPB-07 and MPB-91 induced strong vasorelaxation in IPA rings whether endothelium-denuded or not (figs 5 and $6 \mathrm{~b}$ and figs 5 and $6 a$, respectively). 

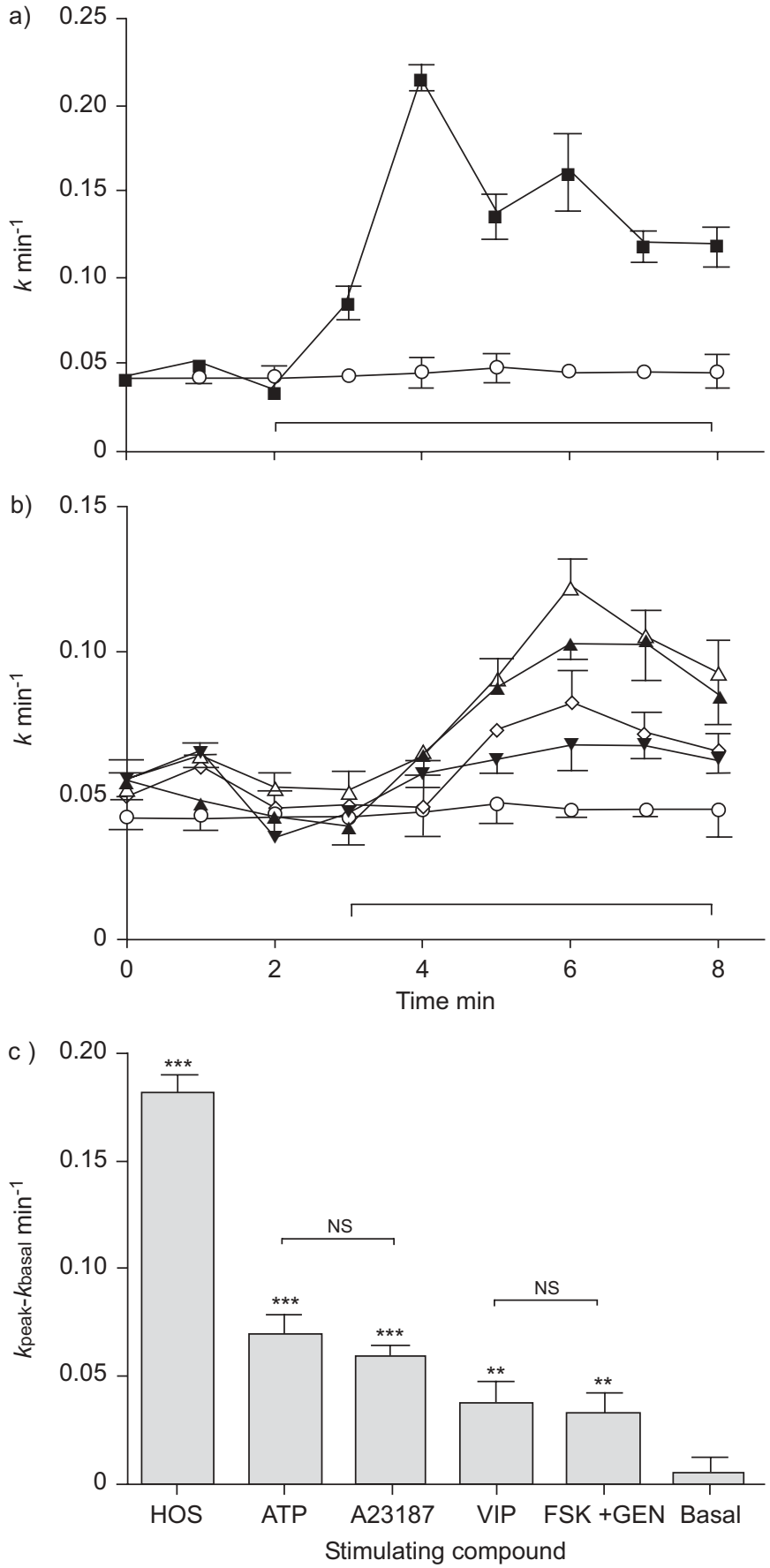

FIGURE 3. Analysis of chloride transport in cultured rat intrapulmonary arterial smooth muscle cells. Stimulation of iodide efflux with time for: a) a hypo-osmotic solution (HOS) (150 mOsm. $\left.\mathrm{L}^{-1} ; \mathbf{D}\right)$; and b) calcium-related compounds $(\mathbf{\Lambda}: 1 \mu \mathrm{M}$ A23187; $\triangle: 100 \mu \mathrm{M}$ adenosine triphosphate (ATP)) and cyclic adenosine monosphosphate agonists ( $\mathbf{\nabla}: 10 \mu \mathrm{M}$ forskolin (FSK) plus $30 \mu \mathrm{M}$ genistein (GEN); $\diamond: 500 \mathrm{nM}$ vasoactive intestinal peptide (VIP)). Horizontal bars represent period during which drugs were present in bath ( $\bigcirc$ : iso-osmotic vehicle alone). $c)$ Maximal time-dependent rate ( $k$; from $a$ and $b)$. Data are presented as mean \pm SEM ( $n=4$ for each) with the basal rate subtracted from the peak rate (kpeak-kbasal). NS: nonsignificant. **: $p<0.01 ; * * *: p<0.001$ versus basal.

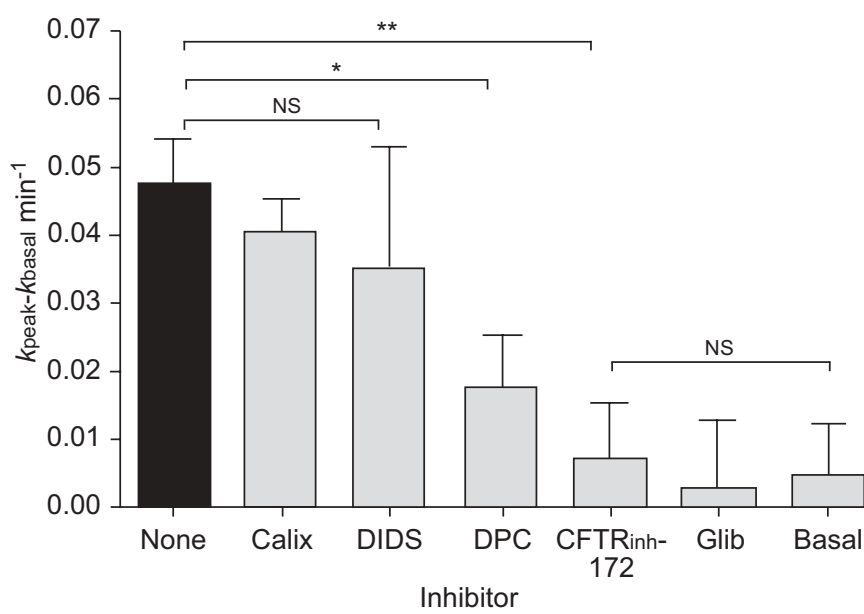

FIGURE 4. Pharmacological inhibition of iodide efflux stimulated by forskolin plus genistein. Cultured smooth muscle cells were stimulated by CAMP agonists (10 $\mu \mathrm{M}$ forskolin plus $30 \mu \mathrm{M}$ genistein) in the presence or absence of $100 \mathrm{nM}$ calixarene (Calix), $500 \mu \mathrm{M}$ 4,4'-diisothiocyanostilbene-2,2'-disulphonate (DIDS), $500 \mu \mathrm{M}$ diphenylamine-2-carboxylic acid (DPC), $10 \mu \mathrm{M}$ CFTR inhibitor (CFTRinh)172 or $100 \mu \mathrm{M}$ glibenclamide (Glib). Data are presented as mean \pm SEM ( $n=4$ for each set of experiments) with the basal time dependent rate subtracted from the peak rate (kpeak-kbasal). Basal: vehicle alone; Ns: nonsignificant. *: $p<0.05$ $* *: p<0.01$

In the presence of $500 \mu \mathrm{M}$ DIDS, a ICl,Ca blocker [11] that had no effect on the cAMP-dependent $\mathrm{Cl}^{-}$transport (fig. 4), $200 \mu \mathrm{M}$ MPB-07 still induced strong relaxation $(59.9 \pm 4.5 \%$ of original precontraction with PHE $(\mathrm{n}=15)$; data not shown), indicating that the effect of MPB is mainly independent of $\mathrm{ICl}, \mathrm{Ca}$ activity.

Finally, in cultured rat IPA myocytes pretreated with $0.3 \mu \mathrm{M}$ PHE, $250 \mu \mathrm{M}$ MPB-07 and MPB-91 stimulated iodide efflux $(\mathrm{n}=4$; fig. 7), whereas MPB-80 induced low CFTR activation (fig. 7b). As with forskolin/genistein, the iodide efflux stimulated by MPB-91 was insensitive to $100 \mathrm{nM}$ calixarene and $500 \mu \mathrm{M}$ DIDS, but was fully inhibited in the presence of $100 \mu \mathrm{M}$ glibenclamide, $500 \mu \mathrm{M}$ DPC (data not shown) and $10 \mu \mathrm{M}$ CFTRinh-172 (fig. 7). CFTRinh-172 also inhibited the response to MPB-07 and MPB- 80 (fig. $7 b$ ).

Taken together, the present results show that activation of the CFTR induces endothelium-independent vasorelaxation in rat IPAs, suggesting a potential and unexpected role of the CFTR in pulmonary vascular tone.

\section{DISCUSSION}

The current report presents evidence that a functional CFTR is endogenously expressed in IPA smooth muscle. First, it was shown that agonists of the cAMP pathway, such as forskolin, stimulated CFTR $\mathrm{Cl}^{-}$channel activity. Secondly, the pharmacology of the CFTR is very similar to that of the epithelial CFTR, in terms of both activation (using MPB derivatives and genistein) and inhibition (using glibenclamide, DPC and CFTRinh-172). Moreover, the structural and pharmacological specificity of MPBs (i.e. the different activity of MPB-80, MPB07 and MPB-91) are similar in IPA cells from rat and mouse aortas $[6,7]$ and in epithelia [21]. Thirdly, activation of the 

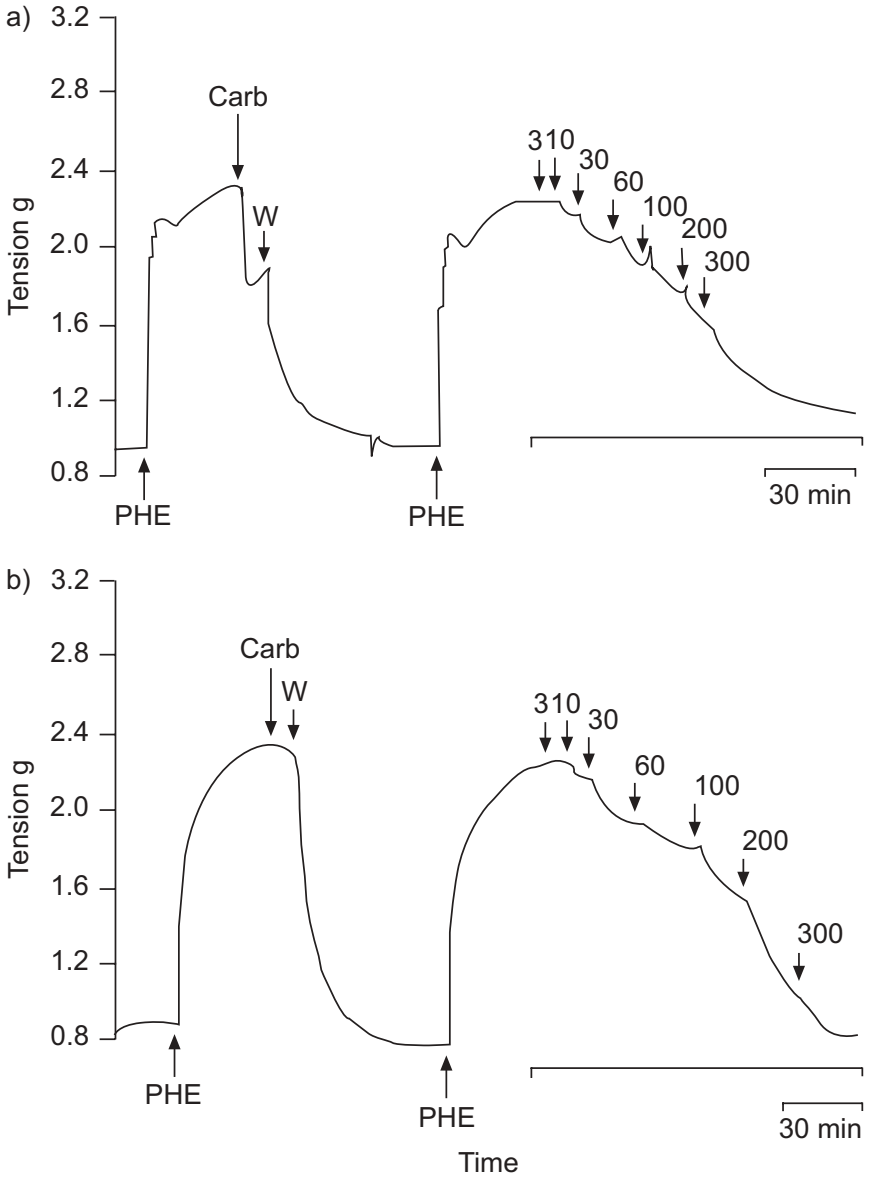

FIGURE 5. Role of the endothelium in the mechanical effect of cystic fibrosis transmembrane conductance regulator activators in rat intrapulmonary arteries (IPAs) in the presence (a) and absence (b) of the endothelium. Typical traces showing isometric tension measurements as a function of time in IPA rings precontracted with $0.3 \mu \mathrm{M}$ phenylephrine (PHE). Bath application of $10 \mu \mathrm{M}$ carbamylcholine (Carb) demonstrated the presence or otherwise of the endothelium. After washout $(\mathrm{W})$, the tension returned to basal levels and $0.3 \mu \mathrm{M}$ PHE was again added. The effect of stepwise increases in the benzoquinolizinium derivatives MPB-07 (a) and -91 (b) concentration were determined in these precontracted vessels (figures above vertical arrows indicate micromolar concentrations). Horizontal bars indicate presence of benzoquinolizinium derivative.

CFTR in IPA leads to endothelium-independent vasorelaxation. The present study is the first to show the presence and function of CFTR channels in primary cultured PASMCs and their implications for pulmonary vasorelaxation.

The presence of $I \mathrm{Cl}, \mathrm{Ca}$ and $I \mathrm{Cl}$,swell in rat PASMCs was also confirmed, and the use of cultured IPA SMCs to study $\mathrm{Cl}^{-}$ transport consequently validated.

The presence of functional cAMP-dependent $\mathrm{Cl}^{-}$channels has previously been suggested in bovine pulmonary arterial smooth muscle by the use of rather nonspecific $\mathrm{Cl}^{-}$channel blockers, such as phenylanthranilic acid, 9-anthracene carboxylic acid, 5-nitro-2-(3-phenylpropylamino)benzoic acid (NPPB) and DIDS, on cAMP-dependent responses induced by 5 -hydroxytryptamine $[17,27]$. The molecular origin of this
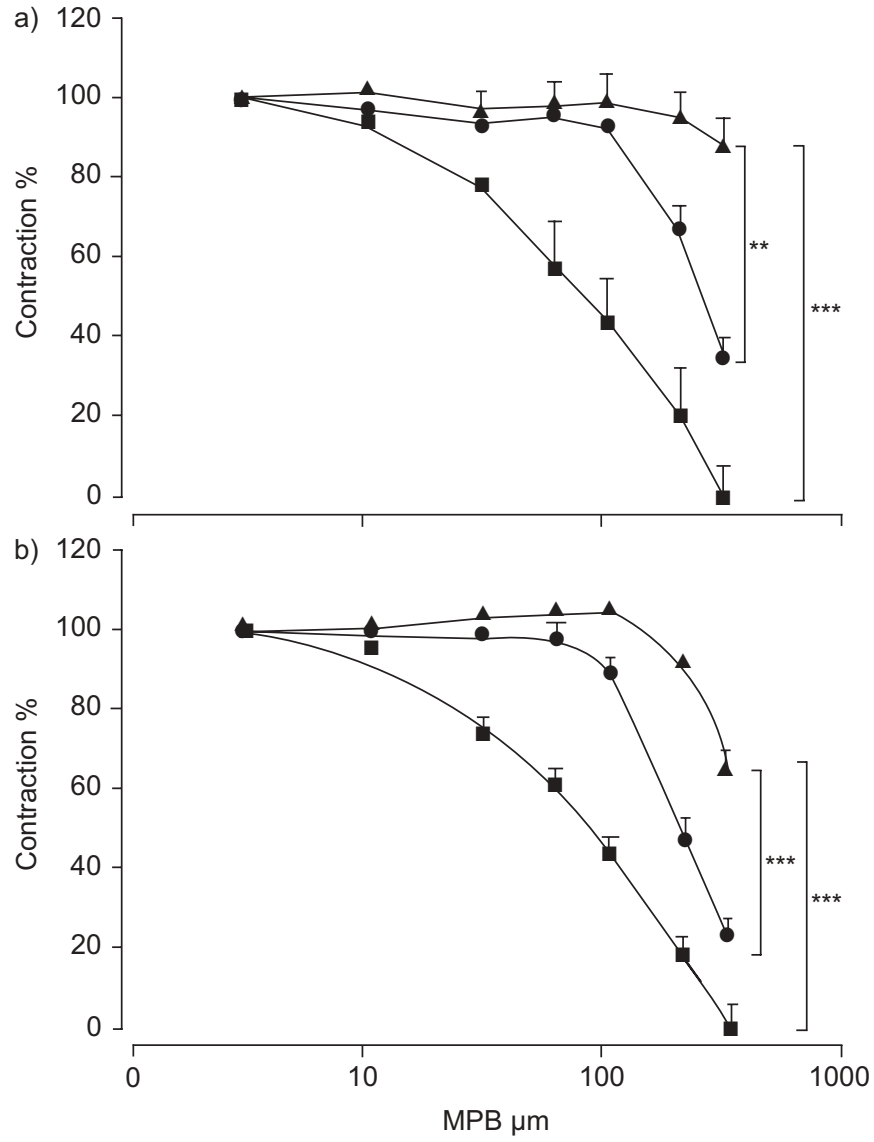

FIGURE 6. Concentration-dependent vasorelaxation of cystic fibrosis transmembrane conductance regulator activators ( $\bullet$ : the benzoquinolizinium derivative MPB-07; $\mathbf{\square}$ : MPB-91; $\mathbf{\Delta}$ : MPB-80) in rat intrapulmonary arteries in: a) the presence; and $b)$ the absence of endothelium. Data are presented as mean \pm SEM $(n=9-15$ rings (a) and 8-14 rings (b)) percentage of original precontraction with $0.3 \mu \mathrm{M}$ phenylephrine. ${ }^{* *}: \mathrm{p}<0.01 ;{ }^{* * *}: \mathrm{p}<0.001$.

channel has not been identified, although the cAMP-related 5hydroxytryptamine responses were inhibited by phenylanthranilic acid and 9-anthracene carboxylic acid but not by DIDS and NPPB. The CFTR is insensitive to DIDS [23], and this result was confirmed in the present study, suggesting that the channel observed in rat IPA may be similar to that observed in bovine pulmonary arterial smooth muscle [17]. The pharmacological profile of CFTR activation in IPA using MPB activators (MPB-91 $>$ MPB-07 $>$ MPB-80) studied with the iodide efflux and isometric tension techniques (in the present report) is similar to that previously obtained in rat or mouse aortic myocytes [6, 7], epithelial cells [21] and tracheal myocytes [5]. Moreover, endothelium removal did not influence pulmonary arterial vasorelaxation in response to MPBs, a result in agreement with that previously reported for rat and mouse aorta [6, 7]. Finally, the activation of CAMP- and MPBdependent iodide efflux was fully inhibited by the CFTRspecific inhibitor CFTRinh-172 in IPA, as in epithelial cells [9] and aortic SMCs [6]. All of these results contributed to the conclusion that the CFTR, as a cAMP-dependent $\mathrm{Cl}^{-}$channel expressed in PASMCs, is involved in endothelium-independent pulmonary vasorelaxation. 

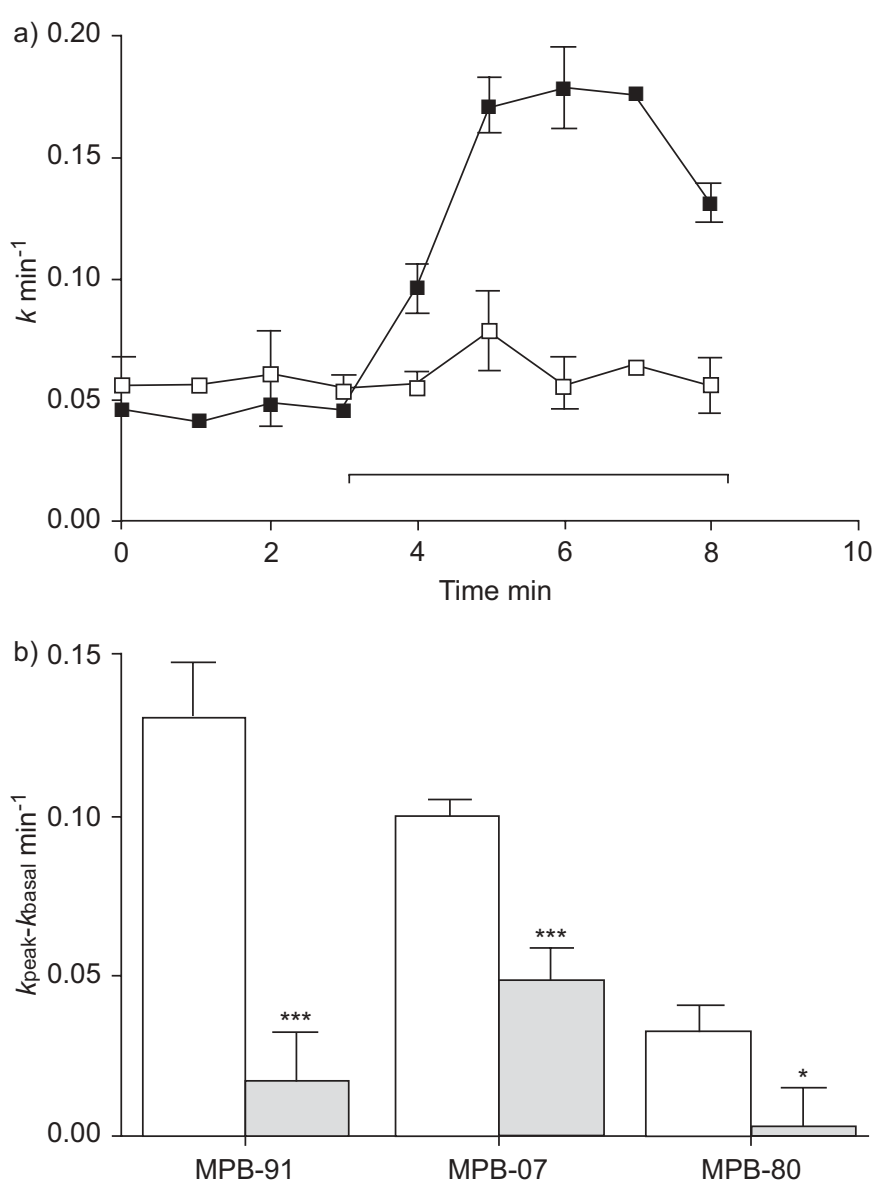

FIGURE 7. Effect of benzoquinolizinium derivative activators of the cystic fibrosis transmembrane conductance regulator (CFTR) on iodide efflux. a) lodide efflux evoked by $250 \mu \mathrm{M}$ MPB-91 in the presence $(\square)$ or absence $(\boldsymbol{\square})$ of $10 \mu \mathrm{M}$ CFTR inhibitor (CFTRinh)-172 as a function of time. Horizontal bar indicates bath application of the drug. b) Effect of $10 \mu \mathrm{M}$ CFTRinh-172 on the MPB-stimulated efflux in the absence $(\square)$ or presence $(\square)$ of CFTRinh-172. All experiments were performed in the presence of $0.3 \mu \mathrm{M}$ phenylephrine. Data are presented as mean \pm SEM $(n=4$ for each) with the basal time dependent rate subtracted from the peak rate (kpeak-kbasal). *: $p<0.05$; ${ }^{* *}$ : $p<0.001$ versus absence of CFTRinh-172 (unpaired t-test).

In epithelial cells, cAMP agonists stimulate transepithelial $\mathrm{Cl}^{-}$ transport via phosphorylation and opening of apical CFTR channels $[1,28]$. In SMCs from the systemic circulation, i.e. aortas [6, 7], or pulmonary circulation (present study), activation of the CFTR was evidenced after precontraction of the muscle cells. These observations are consistent with previous studies showing that cAMP is implicated in the relaxation of vascular SMCs in response to vasodilators such as $\beta$-adrenergic agonists or vasoactive intestinal peptide $[25,26]$. Since the current study demonstrated the presence of the CFTR in IPA, it may explain, at least in part, why an increase in cAMP concentration would induce pulmonary vasorelaxation via activation of the CFTR.

It is noteworthy that the present study was conducted in IPAs, which thus exhibit a functional CFTR. It is tempting to hypothesise that altered CFTR function in pulmonary arteries may be linked to the development of pulmonary hypertension since: 1) IPAs, compared with extrapulmonary arteries, are particularly sensitive to hypoxia and thus strongly involved in the pathogenesis of pulmonary hypertension; 2) patients affected by the genetic disease cystic fibrosis also develop some respiratory diseases, eventually leading to pulmonary hypertension; and 3) some $\mathrm{Cl}^{-}$channels are involved in pulmonary hypertension (see Introduction section). Although this is beyond the scope of the present study, further investigations are required to explore the expression and function of the CFTR in pulmonary arteries from pulmonary hypertensive animals.

In summary, the current report presents the first time direct evidence of functional cystic fibrosis transmembrane conductance regulator expression in rat intrapulmonary arterial primary cultured smooth muscle cells. In a more integrated model, such as intrapulmonary arterial rings, activation of the cystic fibrosis transmembrane conductance regulator induces endothelium-independent vasorelaxation, indicating a potential role of cystic fibrosis transmembrane conductance regulator in the regulation of pulmonary vascular tone. An interesting new therapeutic development could arise from the discovery that cystic fibrosis transmembrane conductance regulator activators are able to relax pulmonary arterial smooth muscle cells, thus being potential anti-hypertensive agents.

\section{ACKNOWLEDGEMENTS}

The authors would like to thank H. Crevel (INSERM U885, University of Bordeaux 2, Bordeaux, France) for technical assistance, C. Vandebrouck (University of Poitiers, Poitiers, France) for cell culture preparation, immunolocalisation of the cystic fibrosis transmembrane conductance regulator and discussions, and V. Thoreau (University of Poitiers) for assistance in RT-PCR and immunoprecipitation experiments.

\section{REFERENCES}

1 Riordan JR, Rommens JM, Kerem B, et al. Identification of the cystic fibrosis gene: cloning and characterization of complementary DNA. Science 1989; 245: 1066-1073.

2 Gadsby DC, Nairn AC. Control of CFTR channel gating by phosphorylation and nucleotide hydrolysis. Physiol Rev 1999; 79: Suppl. 1, S77-S107.

3 Weyler RT, Yurko-Mauro KA, Rubenstein R, et al. CFTR is functionally active in GnRH-expressing GT1-7 hypothalamic neurons. Am J Physiol 1999; 277: C563-C571.

4 Tousson A, Van Tine BA, Naren AP, Shaw GM, Schwiebert LM. Characterization of CFTR expression and chloride channel activity in human endothelia. Am J Physiol 1998; 275: C1555-C1564.

5 Vandebrouck C, Melin P, Norez C, et al. Evidence that CFTR is expressed in rat tracheal smooth muscle cells and contributes to bronchodilation. Respir Res 2006; 7: 113.

6 Robert R, Norez C, Becq F. Disruption of CFTR chloride channel alters mechanical properties and cAMP-dependent $\mathrm{Cl}^{-}$transport of mouse aortic smooth muscle cells. J Physiol 2005; 568: 483-495.

7 Robert R, Thoreau V, Norez C, et al. Regulation of the cystic fibrosis transmembrane conductance regulator channel by $\beta$-adrenergic agonists and vasoactive intestinal 
peptide in rat smooth muscle cells and its role in vasorelaxation. J Biol Chem 2004; 279: 21160-21168.

8 Becq F. On the discovery and development of CFTR chloride channel activators. Curr Pharm Des 2006; 12: 471-484.

9 Ma T, Thiagarajah JR, Yang H, et al. Thiazolidinone CFTR inhibitor identified by high-throughput screening blocks cholera toxin-induced intestinal fluid secretion. J Clin Invest 2002; 110: 1651-1658.

10 Leblanc N, Ledoux J, Saleh S, et al. Regulation of calciumactivated chloride channels in smooth muscle cells: a complex picture is emerging. Can J Physiol Pharmacol 2005; 83: 541-556.

11 Guibert C, Marthan R, Savineau JP. Oscillatory $\mathrm{Cl}^{-}$current induced by angiotensin II in rat pulmonary arterial myocytes: $\mathrm{Ca}^{2+}$ dependence and physiological implication. Cell Calcium 1997; 21: 421-429.

12 Piper AS, Large WA. Multiple conductance states of single $\mathrm{Ca}^{2+}$-activated $\mathrm{Cl}^{-}$channels in rabbit pulmonary artery smooth muscle cells. J Physiol 2003; 547: 181-196.

13 Yuan XJ. Role of calcium-activated chloride current in regulating pulmonary vasomotor tone. Am J Physiol 1997; 272: L959-L968.

14 Dai YP, Bongalon S, Hatton WJ, Hume JR, Yamboliev IA. ClC-3 chloride channel is upregulated by hypertrophy and inflammation in rat and canine pulmonary artery. $\mathrm{Br} \mathrm{J}$ Pharmacol 2005; 145: 5-14.

15 Nakazawa H, Hori M, Murata T, Ozaki H, Karaki H. Contribution of chloride channel activation to the elevated muscular tone of the pulmonary artery in monocrotalineinduced pulmonary hypertensive rats. Jpn J Pharmacol 2001; 86: 310-315.

16 Oriowo MA. Chloride channels and $\alpha_{1}$-adrenoceptormediated pulmonary artery smooth muscle contraction: effect of pulmonary hypertension. Eur J Pharmacol 2004; 506: 157-163.

17 Day RM, Agyeman AS, Segel MJ, et al. Serotonin induces pulmonary artery smooth muscle cell migration. Biochem Pharmacol 2006; 71: 386-397.
18 Zhao YJ, Wang J, Rubin LJ, Yuan XJ. Roles of $\mathrm{K}^{+}$and $\mathrm{Cl}^{-}$ channels in cAMP-induced pulmonary vasodilation. Exp Lung Res 1998; 24: 71-83.

19 Fiedler MA, Nemecz ZK, Shull GE. Cloning and sequence analysis of rat cystic fibrosis transmembrane conductance regulator. Am J Physiol 1992; 262: L779-L784.

20 Pauvert O, Bonnet S, Rousseau E, Marthan R, Savineau JP. Sildenafil alters calcium signaling and vascular tone in pulmonary arteries from chronically hypoxic rats. Am J Physiol Lung Cell Mol Physiol 2004; 287: L577-L583.

21 Marivingt-Mounir C, Norez C, Derand R, et al. Synthesis, SAR, crystal structure, and biological evaluation of benzoquinoliziniums as activators of wild-type and mutant cystic fibrosis transmembrane conductance regulator channels. J Med Chem 2004; 47: 962-972.

22 Pourageaud F, Leblais V, Bellance N, Marthan R, Muller B. Role of $\beta_{2}$-adrenoceptors ( $\beta$-AR), but not $\beta_{1^{-}}, \beta_{3^{-}}$AR and endothelial nitric oxide, in $\beta$-AR-mediated relaxation of rat intrapulmonary artery. Naunyn Schmiedebergs Arch Pharmacol 2005; 372: 14-23.

23 Singh AK, Venglarik CJ, Bridges RJ. Development of chloride channel modulators. Kidney Int 1995; 48: 985-993.

24 Nilius B, Droogmans G. Amazing chloride channels: an overview. Acta Physiol Scand 2003; 177: 119-147.

25 Barnes PJ, Liu SF. Regulation of pulmonary vascular tone. Pharmacol Rev 1995; 47: 87-131.

26 Savineau JP, Gonzalez De La Fuente P, Marthan R. Effect of vascular smooth muscle relaxants on the protein kinase $\mathrm{C}$ mediated contraction in the rat pulmonary artery. Eur J Pharmacol 1993; 249: 191-198.

27 Lee SL, Fanburg BL. Serotonin produces a configurational change of cultured smooth muscle cells that is associated with elevation of intracellular cAMP. J Cell Physiol 1992; 150: 396-405.

28 Schwiebert EM, Benos DJ, Egan ME, Stutts MJ Guggino WB. CFTR is a conductance regulator as well as a chloride channel. Physiol Rev 1999; 79: Suppl. 1, S145S166. 